\title{
Criterios en la selección de pacientes para optimizar resultados en liposucción asistidda por láser de abdomen y flancos: análisis de 340 cirugías
}

\section{Patient selection criteria to optimize results in laser-assisted liposuction of the abdomen and flanks: analysis of 340 surgeries}

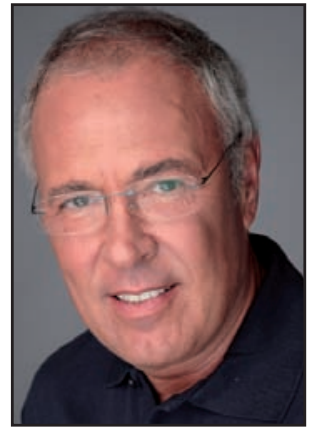

Moreno-Moraga J.

\author{
Moreno-Moraga J.*, Alcolea JM. ${ }^{\star *}$, Royo de la Torre J. ${ }^{\star \star *}$, Bravo E. ${ }^{\star \star \star *}$,

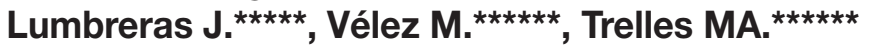

\section{Resumen}

El objetivo de nuestro estudio es valorar mediante ecografía y análisis estadístico la respuesta a la liposucción asistida por láser en abdomen y flancos Para ello, se tuvo en cuenta la disminución del tejido adiposo, la retracción cutánea y el grado de satisfacción de las pacientes con los resultados.

Realizamos un estudio retrospectivo sobre 340 cirugías en pacientes multíparas mayores de 40 años, con o sin diástasis de rectos, que rechazaron la abdominoplastia con lipectomía. Las intervenciones se efectuaron mediante liposucción asistida por dos láseres de Diodo, en actuación simultánea, que emitían respectivamente en 924 y $975 \mathrm{~nm}$, bajo sedo-analgesia y con anestesia tumescente. Los láseres se programaron para emisión en modo continuo, actuando a unísono con potencia de $20 \mathrm{~W}$ hasta alcanzar un promedio de entre 10 a $12 \mathrm{~kJ}$ de energía acumulada para los flancos, y de entre 12 a $18 \mathrm{~kJ}$ para el abdomen.

La valoración subjetiva y objetiva se realizó mediante escala GAIS (Global Aesthetic Improvement Scale). Se tomaron fotografías antes, 1 y 6 meses después de la intervención. En abdomen se realizaron ecografías antes y 6 meses después de la cirugía, y se empleó t de Student como test de contraste para el estudio analítico. En 60 pacientes seleccionados aleatoriamente como muestra representativa se realizaron fotografías digitales de la superficie de la piel a fin de valorar comparativamente por programa de ordenador la texturaflacidez de la condición del tejido antes, 1 y 6 meses después de la cirugía.

En ninguno de los resultados se observó isquemia y/o quemaduras. La disminución del tejido adiposo, según las ecografías, fue estadísticamente significativa $(\mathrm{p}<0,05)$ comparativamente a los 6 meses del tratamiento. La valoración de las fotografías antes y 6 meses después de la cirugía por un médico ajeno a estudio, al igual que la textura-flacidez cutánea, fue Buena o Muy Buena. La valoración subjetiva por cuestionarios de escala GAIS alcanzó un 57,1\% (194 pacientes) al sumar los resultados Buenos y Muy Buenos. En cambio, la valoración objetiva por el médico fue sensiblemente superior, un $74 \%$ (252 pacientes).

En conclusión, según los estudios realizados creemos que la liposucción asistida por láser es una técnica eficaz y reproducible, que obtiene notables resultados cuando las pacientes presentan depósitos de grasa localizada y no sufren diástasis de los rectos abdominales como consecuencia de partos múltiples. También es importante que, de forma ideal, las pacientes no presenten un índice de masa grasa corporal (BMI) superior a 26 para predecir y obtener óptimos resultados.
Palabras clave Liposucción, Liposucción asistida por láser, Láser lipólisis.
IV
Ultrasound and statistical analysis of results of laser-assisted liposuction of the abdomen and flanks have been evaluated. Decrease in fat tissue, skin retraction and patient satisfaction with results have all been taken into consideration.

This retrospective study corresponds to 340 surgeries, done on multiparous patients over 40 years age, with and without abdominal recti muscle diastasis that rejected going through abdominal lipectomy. Surgeries were carried out by laser assisted liposuction with two Diode lasers emitting at 924 and $975 \mathrm{~nm}$, operating simultaneously, performed under sedation and analgesia, and injecting tumescent anaesthesia. Lasers were programmed for emission in continuous mode with both systems operating at a power of $20 \mathrm{~W}$, reaching a total mean accumulated energy of between 10 and $12 \mathrm{~kJ}$ for flanks, and between 12 and $18 \mathrm{~kJ}$ for the abdomen.

Subjective and objective evaluation was carried out using a GAIS scale (Global Aesthetic Improvement Scale). Photographs were taken before, 1 and 6 months after procedures. For the abdomen ultrasound examination were done before and 6 months after surgery, and Student's t-test was implemented for the statistical analysis. In 60 patients, randomly chosen as a representative sample, digital photographs of the skin surface were taken in order to comparatively evaluate skin texture-laxity condition, before, 1 and 6 months after surgery with the aid of a computer programme.

No ischemia or burns were observed in any patient. According to ultrasound exam, decrease in fat tissue was statistically significant $(p<0,05)$ when compared to 6 months after surgery. Evaluation by an independent doctor to the study, before and 6 months after photographs as well as the skin texture-laxity, were Good and Very Good. Subjective results obtained through questionnaires using GAIS scale reached 57,1\% (194 patients) satisfied with results, totalling the Good and Very Good results. However, the objective evaluation, done by the Doctor, was significantly better regarding results obtained, 74\% (252 patients).

In conclusion, laser assisted liposuction is an effective and reproducible technique that obtains notable results when patients presented localized fat deposits and did not suffer diastasis due to multiple pregnancies. It is also important that, ideally, patients selected should not have a BMI of over 26 in order to obtain optimal results.

*** Licenciada en Medicina y Cirugía, Instituto Médico Láser. Madrid, España.

**** Cirujano Plástico, Instituto Médico Láser, Madrid, España.

***** Anestesista, Instituto Médico Láser, Madrid, España.

****** Dermatólogo, Instituto Médico Vilafortuny, Cambrils, Tarragona, España.

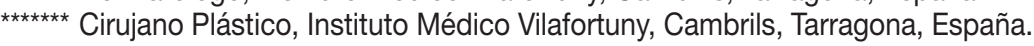




\section{Introducción}

La liposucción asistida por láser es un procedimiento bien establecido en la actualidad, según muestran las diversas publicaciones que se encuentran en la bibliografía internacional (1-4). La técnica operatoria tiene ventajas sobre la liposucción convencional en cuanto a la contracción de la piel que se obtiene por el efecto térmico que produce la absorción de la energía láser en el tejido (5). Igualmente, varios autores parecen estar de acuerdo en 2 conclusiones notables: 1 . La técnica láser obtiene mejores resultados en las adiposis localizadas, siendo su eficacia estética menos notable cundo se trata de eliminar grandes volúmenes de grasa en zonas corporales extensas; 2 . La mejor valoración de los resultados que pueden conseguirse con la técnica láser debe realizarse al menos 6 meses después de la cirugía (6-8). Pero estas 2 conclusiones objetivas determinadas por la experiencia del cirujano, no siempre van de la mano de las opiniones de las pacientes, para las cuales cuenta mucho más el aspecto estético al que dan más importancia, que los indiscutibles efectos de contracción de la piel, siendo ésta última la que contribuye de forma notable a la remodelación de las áreas intervenidas (9).

El presente estudio retrospectivo analiza objetiva y subjetivamente los efectos en 340 pacientes, todas ellas mujeres multíparas de edad superior a 40 años, en las que se practicó una sola intervención de liposucción asistida por láser en abdomen y flancos.

\section{Material y método}

Realizamos un estudio retrospectivo para analizar los resultados obtenidos mediante liposucción asistida por láser en mujeres que rechazaron la intervención de abdominoplastia con lipectomía. Revisamos un total de 450 historias clínicas de pacientes intervenidas entre 2009 y 2013, con el permiso del comité ético de la institución donde se realizaron las cirugías. Cada historia incluía consentimiento informado, cuestionarios de satisfacción, fotografías, ecografías para definir los cambios en el espesor del tejido graso subcutáneo, y 60 exámenes de la superficie de la piel con cámara fotográfica digital de superficie y análisis por ordenador. También se controló el estado de la musculatura del abdomen como factor a tener en cuenta en el momento del pronóstico y evaluación de los resultados. En el consentimiento informado se hizo especial hincapié en comunicar que la diástasis de rectos no mejoraría mediante la intervención láser.

Los criterios de inclusión para seleccionar la muestra final fueron: pacientes de sexo femenino, edad superior a 40 años, que al menos hubieran tenido 2 embarazos, que manifestaran rechazo total a ser intervenidas de abdominoplastia y/o lipectomía, aunque este fuera el procedimiento quirúrgico de primera indicación. Como criterio de exclusión y puesto que todas las historias tenían anotado el BMI de las pacientes, seleccionamos el que no fuera $>30$.

Obtuvimos así un total de 340 pacientes, con una media de edad de 52,3 $\pm 5,61$ (entre 41 a 69 años). Todas las pacientes habían tenido entre 2 y 3 embarazos (media $2,4 \pm 0,48)$. Se prestó especial atención al número de cesáreas realizadas: a 89 pacientes $(26,2 \%)$ se les había practicado 1 cesárea; 47 pacientes $(13,8 \%)$ tuvieron 2 cesáreas; y 5 pacientes $(1,5 \%)$ tuvieron 3 cesáreas. En 199 pacientes $(58,5 \%)$ no había historia de intervención quirúrgica previa. Las características de las pacientes se presentan en la Tabla I.

\section{Sistema láser y dosimetría}

Para las cirugías se había empleado un sistema láser dual, de 2 Diodos integrados en la misma consola (Aspire SlimLipo, Palomar ${ }^{\circledR}$ Burlington, MA, EE.UU.). Uno de los láseres emitía a $924 \mathrm{~nm}$ y el otro a $975 \mathrm{~nm}$. Los láseres en este sistema pueden utilizarse de forma individual o simultánea. También se puede controlar de forma independiente la emisión en modo continuo y el nivel de potencia deseado. En el protocolo de tratamiento se había fijado acumular una determinada energía por área de tratamiento, a fin de hacer reproducible la dosificación láser

Tabla I. Características de la muestra y resultados según distintas variables y mediciones.

\begin{tabular}{|c|c|c|c|c|c|c|c|c|c|c|c|c|}
\hline \multirow{2}{*}{$\begin{array}{l}\text { Grupos } \\
\text { etarios }\end{array}$} & \multirow{2}{*}{ Edad } & \multirow{2}{*}{$\begin{array}{l}N^{0} \text { por } \\
\text { grupo }\end{array}$} & \multirow{2}{*}{$\mathrm{BMI} \pm \mathrm{DE}$} & \multirow{2}{*}{$\begin{array}{l}\mathrm{N}^{0} \text { hijos } \\
\pm \mathrm{DE}\end{array}$} & \multicolumn{2}{|c|}{$\begin{array}{c}\text { Ecografía } \\
\text { Grasa en cm } \pm \mathrm{DE}\end{array}$} & \multirow{2}{*}{$\begin{array}{c}\text { Diástasis } \\
\text { rectos } \\
\text { cm } \pm \mathrm{DE}\end{array}$} & \multicolumn{3}{|c|}{\begin{tabular}{|c|} 
Textura-flacidez cutánea (N 60) \\
Cámara digital de superficie
\end{tabular}} & \multirow{2}{*}{$\begin{array}{c}\text { GAIS } \\
\text { pacientes }\end{array}$} & \multirow{2}{*}{$\begin{array}{c}\text { GAIS } \\
\text { Doctor }\end{array}$} \\
\hline & & & & & Antes & 6 meses & & Antes & $1 \mathrm{mes}$ & 6 meses & & \\
\hline 1 & $40-47$ & 82 & $26,4 \pm 1,87$ & $2,4 \pm 0,48$ & $4,2 \pm 0,72$ & $1,4 \pm 0,25$ & $1,8 \pm 1,28$ & $1,2 \pm 0,76$ & $2,2 \pm 0,74$ & $3,1 \pm 0,75$ & $2,9 \pm 1,15$ & $3,5 \pm 1,02$ \\
\hline 2 & $48-55$ & 137 & $25,7 \pm 1,98$ & $2,3 \pm 0,51$ & $4,4 \pm 0,67$ & $1,6 \pm 0,23$ & $2,2 \pm 1,37$ & $1,2 \pm 0,78$ & $2,1 \pm 0,75$ & $3,2 \pm 0,72$ & $2,8 \pm 1,09$ & $3,2 \pm 1,03$ \\
\hline 3 & $56-69$ & 121 & $26,1 \pm 2,01$ & $2,3 \pm 0,42$ & $4,1 \pm 0,89$ & $1,3 \pm 0,23$ & $2,5 \pm 1,18$ & $1,1 \pm 0,81$ & $2,1 \pm 0,71$ & $3,0 \pm 0,71$ & $2,6 \pm 1,11$ & $3,1 \pm 1,05$ \\
\hline
\end{tabular}


total de la cirugía según el área intervenida. Se empleó una cánula de $1,5 \mathrm{~mm}$ de diámetro y $27 \mathrm{~cm}$ de longitud, a través de la cual se pasó la fibra de $600 \mu \mathrm{m}$ del láser, dejando asomar $3 \mathrm{~mm}$ de la fibra en la punta de la cánula. Ambos láseres se programaron a $20 \mathrm{~W}$ de potencia en modo continuo para el tratamiento de todas las pacientes. La temperatura externa del área de tratamiento se controló con un termómetro de infrarrojos (CEM DT-880B, Shenzhen Everbest Machinery Industry $\mathrm{Co}^{\circledR}$. Ltd, China), considerándose suficiente y seguro no superar $\operatorname{los} 42^{\circ} \mathrm{C}$.

\section{Marcaje preoperatorio}

La línea superior en la vista frontal se marcó siguiendo el surco mamario inferior y se trazó una línea accesoria a nivel del reborde costal en aquellos casos en los que existía acumulación de grasa en dicha zona. La línea inferior se marcó siguiendo el relieve de los huesos pelvianos. En esta zona se hizo marcación topográfica de los relieves de grasa más prominentes.

En la parte posterior el marcado se extendió hasta la columna vertebral. En la parte ínfero-posterior se marcó siguiendo la línea de transición entre el flanco y la región glúteo-trocantérea. La marcación topográfica de los distintos relieves de las acumulaciones de grasa se realizó siguiendo el patrón tradicional.

\section{Anestesia}

La intervención en todas las pacientes se realizó bajo sedación y analgesia monitorizada, teniendo en cuenta los antecedentes personales y análisis clínicos personalizados. Las pacientes estaban en ayunas de 8 horas para sólidos y 6 horas para líquidos. En el quirófano se procedió a la monitorización con ECG, tensión arterial, gasometría y ventilación mediante gafas nasales, y constante medición de la presión parcial de $\mathrm{CO}_{2}$ (capnografía).

A todas las pacientes se les tomó una vía con catéter 18G y se les realizó profilaxis antibiótica con amoxicilina/ácido clavulánico 875/125 mg. Para prevenir las náuseas se les administró dexametasona $(0,1 \mathrm{mg} / \mathrm{kg})$, droperidol 1,25 mg y ondansetrón $4 \mathrm{mg}, 1$ hora antes de la anestesia.

La sedación por anestesista se realizó con midazolam 1 a $2 \mathrm{mg}$, y analgesia con remifentanilo 0,02-0,04 $\mathrm{mcg} / \mathrm{kg} / \mathrm{min}$, comenzando 5 minutos antes de proceder a la infiltración tumescente con solución de Klein en las zonas previamente marcadas. La solución de Klein se preparó con $1 \mathrm{~L}$ de suero salino al que se adicionaron $50 \mathrm{ml}$ de lidocaína al 1\%,1 ml de adrenalina 1:1.000.000 y $2,5 \mathrm{ml}$ de bicarbonato de sodio al 8,4\% $(10,11)$.

La reposición de líquidos se realizó a las dosis habituales teniendo en cuenta los requerimientos básicos más las pérdidas estimadas, tras un control estricto de las cantidades totales infiltradas y del volumen final aspirado.

Tras la intervención, las pacientes pasaron a recuperación donde se continuó con control de constantes y administración de cloruro de sodio, con mantenimiento de la vía. Trascurrida la primera hora se probó la tolerancia a líquidos, dando de alta a las pacientes a las 2 horas de la cirugía.

En el postoperatorio se recomendó tratamiento con paracetamol $1 \mathrm{gr} / 6$ horas y prednisolona $15 \mathrm{mg} / \mathrm{día}$, ambos durante 5 días, pudiendo prolongar el tiempo de tratamiento si fuera necesario.

\section{Técnica quirúrgica}

La irradiación de la energía láser se realizó con empujes de la cánula hacia delante y atrás con movimientos en abanico desde los puntos de entrada previamente marcados. Se trataron primero los planos profundos del tejido graso, y posteriormente los medios y superficiales. Todas las pacientes recibieron un total de energía global calculada en función de las regiones tratadas, como sigue: flancos entre 10 a 12 kilojulios (kJ); región superior del abdomen entre 12 a $15 \mathrm{~kJ}$; y región inferior del abdomen entre 15 a $18 \mathrm{~kJ}$. En consecuencia, los promedios totales variaron de 35 a $45 \mathrm{~kJ}$ para las 2 áreas dependiendo del volumen de grasa que presentaba el tejido y en función del BMI de la paciente.

Para llevar a cabo la liposucción, una vez efectuado el paso de la irradiación láser, se emplearon cánulas tipo Mercedes de $3 \mathrm{~mm}$, conectadas a un aparato de 1,5 bar de presión negativa (Lipo-MR ${ }^{\circledR}$, Ordisi SA, Barcelona, España). Se tuvo en cuenta que el volumen total de aspiración excediera el $70 \%$ del volumen de la infiltración tumescente.

En el postoperatorio no se realizaron tratamientos adicionales, como drenaje linfático, endermología o radiofrecuencia.

A todas las pacientes se les indicó llevar una faja compresiva tipo Reston ${ }^{\circledR}$, durante 15 días. Esta faja incluye una espuma siliconada en lámina de $2 \mathrm{~cm}$ de grosor que se dispone en contacto con el abdomen. Las pacientes fueron visitadas una semana después. Se valoró la presencia de seromas, fibrosis e irregularidades en las áreas intervenidas, así como cualquier otro defecto. Las siguientes visitas de control fueron 1 y 6 meses después de la intervención.

\section{Valoración ecográfica}

A todas las pacientes se les practicó ecografía del área abdominal (Esaote ${ }^{\circledR}$ MyLab25Gold, Italia), en posición erecta, antes y 6 meses después de la cirugía. Se determinó el espesor del panículo adiposo y la presencia o no de diástasis de los músculos rectos abdominales. La sonda ecográfica se colocó $3 \mathrm{~cm}$ por encima del ombligo. La diástasis se clasificó en 3 grados atendiendo a la medida obtenida por ecografía de la separación de los músculos rectos abdominales. La diástasis severa, igual o $>3 \mathrm{~cm}$, estuvo presente en 148 pacientes $(43,53 \%)$; diástasis moderada, de 1 a $3 \mathrm{~cm}$, en 162 (47,65\%); y diástasis no significativa, $<1 \mathrm{~cm}$, en $30(8,82 \%)$.

Se asumió que la diástasis de los rectos abdominales no era susceptible de mejorar y/o corregirse mediante la liposucción asistida por láser y así se les informó a las pacientes. 


\section{Fotografía}

Se obtuvieron fotografías antes y 6 meses después de la cirugía de todas las pacientes. Las fotografías fueron tomadas con la paciente de pie, de frente y de ambos perfiles. Se empleó el sistema Vectra 3D Analysis ${ }^{\circledR}$ (Canfield Scientific, Fairfield, New Jersey, EE.UU).

\section{Valoración subjetiva}

Todas las pacientes valoraron los resultados conseguidos mediante una escala GAIS (Global Aesthetic Improvement Scale) atendiendo a la reducción de volumen, la retracción de la piel y el aplanamiento del abdomen. Según la puntuación otorgada, se consideraron 5 grupos: Grupo I: puntuación 0, resultado Nulo; Grupo II: puntuación 1, resultado Pobre; Grupo II: puntuación 2, resultado Moderado; Grupo III: puntuación 3, resultado Bueno; Grupo 4, puntuación 4, resultado Muy Bueno.

\section{Valoración objetiva}

Se llevó a cabo por un médico familiarizado con los tratamientos de medicina estética corporal y con el procedimiento quirúrgico. Este médico no participó en la selección de la muestra ni había participado en las intervenciones quirúrgicas. En cada seguimiento se anotaron cuidadosamente las incidencias, prestando especial atención a la presencia de quemaduras o hiperpigmentaciones así como al dolor residual y a los cambios de sensibilidad del área tratada. También se valoraron posibles signos de fiebre, seroma, hematoma o edema prolongado. En esta valoración se empleó el mismo tipo de escala GAIS, siguiendo iguales pautas que las empleadas por las pacientes. Por parte del médico se valoraron los datos obtenidos por ecografías de antes y 6 meses después de la intervención.

\section{Análisis de la textura-flacidez cutánea por cámara di- gital de superficie}

En la valoración objetiva de la calidad y contracción cutánea se tuvo en cuenta, en base a los resultados de tratamientos realizados con anterioridad, que 6 meses es el tiempo idóneo para que desaparezca cualquier resto de edema y para que el tejido alcance su total reafirmación $(8,12)$. Asimismo, 6 meses es también el tiempo necesario para que la piel alcance su máxima contracción.

La textura-flacidez de la piel se documentó de forma objetiva capturando imágenes en la misma área donde se realizaron las mediciones ecográficas; es decir, $3 \mathrm{~cm}$ por encima del ombligo, antes, 1 mes y 6 meses después de la cirugía. Para este cometido, las fotografías de la superficie de la piel se realizaron estando la paciente de pie en espiración mantenida. A fin de determinar con seguridad la zona de la toma de las fotografías, se empleó en cada una de las 3 mediciones la misma lámina transparente donde se dibujó con rotulador permanente la forma del ombligo, tomando como punto de referencia anatómica su parte superior, en el centro de la ceja del ombligo. Para la fotografía digital de superficie se empleó la cámara del aparato Antiaging SD ${ }^{\circledR}$ (CliniPro, Barcelona, España), que permite analizar comparativa y estadísticamente las características de conformación de la superficie cutánea y su relieve. Las mediciones en los 3 tiempos de control dedujeron la evolución de la tensión y la acción obtenida por el tratamiento sobre la flacidez de la piel. Esta técnica conocida como anisotropía de relieves permite un análisis acertado de la condición cutánea, midiendo sus propiedades físicas generales que se determinan según la variación que muestra la propagación de la luz. La imagen de topografía tridimensional se graba por triangulación óptica de barrido con una técnica de proyección de vídeo-luz. La imagen digital obtenida, se procesa automáticamente por un programa de ordenador que mide la profundidad y rugosidad, deduciendo tanto la textura superficial como la consistencia y rugosidad de la dermis. Una vez que las imágenes fueron digitalizadas y transferidas al ordenador, se valoraron cuantitativamente obteniendo un cálculo analítico de algoritmos matemáticos (con los que cuenta el programa del ordenador), a fin de reconstruir los datos fotografiados en un perfil tridimensional altamente preciso. La evaluación se traduce entonces en un gráfico de líneas que muestra las características del área examinada, y así se pueden comparar antes y después de la cirugía emplazando paralelamente las imágenes examinadas. El índice de cambio corresponde a la diferencia entre los valores promedio máximos y mínimos, tomando como referencia la distinta profundidad entre los picos y valles que representan la homogeneidad de la piel, y por ende su tensión.

De las 340 pacientes a las que se les realizó cirugía láser, se tomó una muestra aleatoria de 16, 23 y 21 pacientes correspondientes a cada uno de los grupos de edades reflejados en la Tabla I, sumando un total de 60 pacientes. Esta selección aleatoria permitió dar una imagen acertada de los 3 grupos de pacientes que se consideraron en el estudio.

Las puntuaciones otorgadas respecto del estado de la piel, atendiendo a su textura-flacidez, y/o retracción de la misma, fueron: 0=Muy Mal, 1=Mal, 2=Regular, 3=Bien, 4=Muy Bien.

\section{Análisis estadístico}

Se empleó el sistema SPSS v.20 para Windows. Como test de contraste se seleccionó la t de Student para muestras independientes y correlaciones lineales de Pearson, tomando $\mathrm{p}=0,05$ como punto de significación. Los datos se obtuvieron cómo media \pm desviación estándar.

\section{RESULTADOS}

Ninguna de las pacientes presentó complicaciones graves y no se encontraron signos de isquemia, ni quemaduras cutáneas. No hubo alteraciones de la sensibilidad. Tampoco existieron infecciones ni compromiso circulatorio. Los efectos secundarios fueron leves y consintieron en equimosis o pequeños hematomas que 
se reabsorbieron de forma espontánea. El edema, de grado variable, se interpretó como un signo propio del procedimiento debido a la reacción inflamatoria del tejido. Para el control del dolor bastó paracetamol cada 6 horas.

Se pudo objetivar que en el análisis de la texturaflacidez, realizado mediante la cámara digital de superficie, la mejoría de la piel era sensiblemente mejor a los 6 meses de realizado el tratamiento (Fig. 1 y 2).

En la valoración subjetiva mediante escala GAIS, 2 pacientes valoraron los resultados como Nulos $(0,6 \%)$, 61 como Pobres (17,9\%), 83 como Moderados $(24,4 \%)$, 121 como Buenos (35,6\%), y 73 como Muy Buenos $(21,5 \%)$ (Fig. 3). Según las puntuaciones de la escala GAIS el valor promedio de las pacientes fue de 2,7 \pm 1,14. La valoración objetiva por parte del examinador fue: 13 pacientes con resultados Pobres $(3,8 \%), 75$ Moderados $(22,1 \%), 159$ Buenos (46,7\%) y 93 Muy Buenos $(27,3 \%)$. El valor promedio en la escala GAIS fue de 3,2 $\pm 0,92$ (Fig. 4).

Las mediciones ecográficas de la capa de tejido graso, practicadas antes y 6 meses después de la intervención, se contrastaron mediante el test t de Student con un resul- tado de $\mathrm{p}<0,05$ para un intervalo de confianza del $95 \%$. La media en cm de disminución del tejido graso fue de $3,0 \pm 0,49$, lo que supuso una disminución de la capa de tejido graso del $67,3 \%$.

Las correlaciones de Pearson, en función de las variables de edad, número de hijos y número de cesáreas, no fueron significativas $(p>0,05)$. La correlación entre la disminución de tejido graso y la escala GAIS no fue significativa en el caso de las pacientes $(\mathrm{p}>0,05)$, pero si lo fue en la escala GAIS del examinador $(\mathrm{p}<0,05)$, lo que puede explicase porque en la valoración del médico tiene una indudable importancia la medición ecográfica. Las pacientes valoran más el aspecto estético por el examen visual, siendo destacable el hecho de que los peores resultados fueron otorgados por las pacientes que sufrían diástasis de rectos. Hay que tener en cuenta que 148 pacientes $(43,53 \%)$ se clasificaron como diástasis severa, $162(47,65 \%)$ como diástasis moderada, y solo 30 $(8,82 \%)$ como diástasis no significativa. En consecuencia, este dato fue más notable en aquellas pacientes cuya diástasis fue clasificada como severa, independientemente de que la retracción de la piel, medida por cámara digital de superficie, diera un valor máximo.

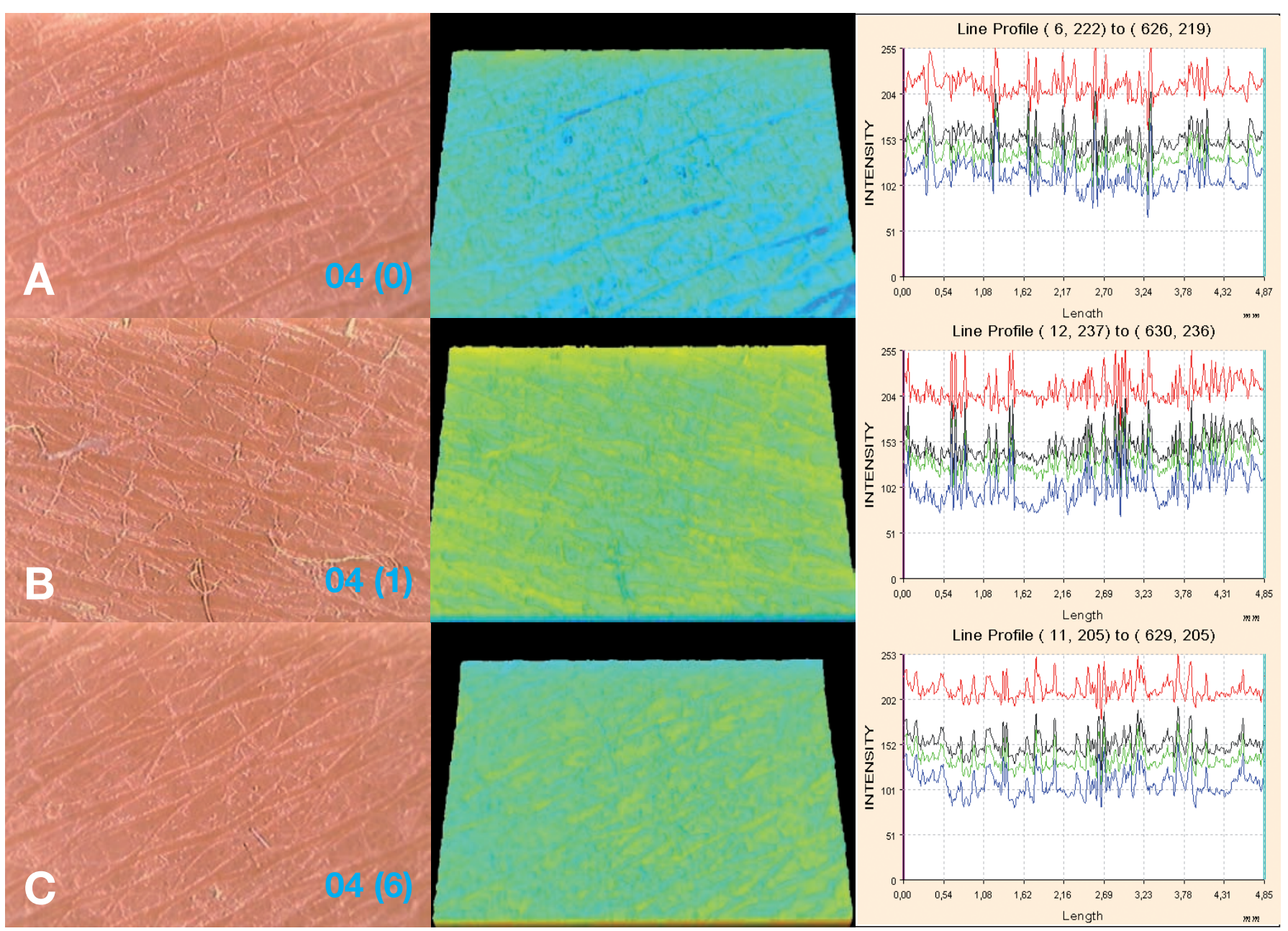

Fig. 1. Detección de la textura/tensión cutánea por cámara digital de superficie en la paciente $n^{\circ} 4$. A: antes de la cirugía; $B$ : 1 mes después de la cirugía, y C: 6 meses después. En las imágenes de la columna izquierda se muestra en superficie la disposición de las líneas de tensión de la piel. Su mayor separación indica flacidez y laxitud cutánea. En la columna central se resalta, en dos dimensiones, la irregularidad del patrón de tensión cutánea. Los gráficos de la derecha representan los valores de las imágenes A, B y C analizadas por el programa de ordenador. Correlativa y comparativamente se muestra de forma objetiva la mejoría que experimenta la piel en su textura-tensión, de acuerdo a las mediciones efectuadas. 


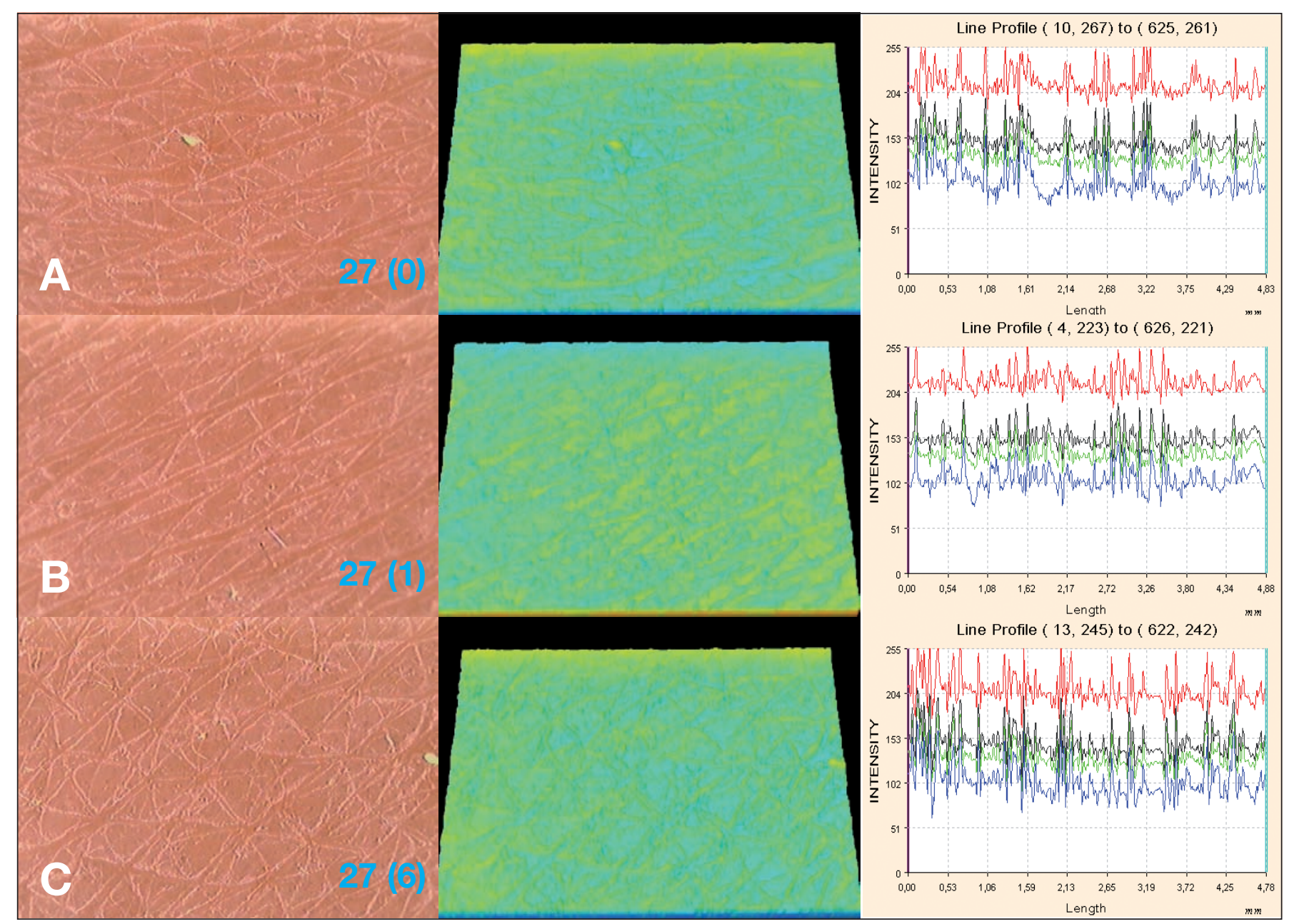

Fig. 2. Paciente $n^{\circ}$ 27. Progreso en la calidad de la tensión y acción contra la flacidez cutánea en las valoraciones efectuadas y de forma comparativa, antes, 1 y 6 meses después de la liposucción asistida por láser.

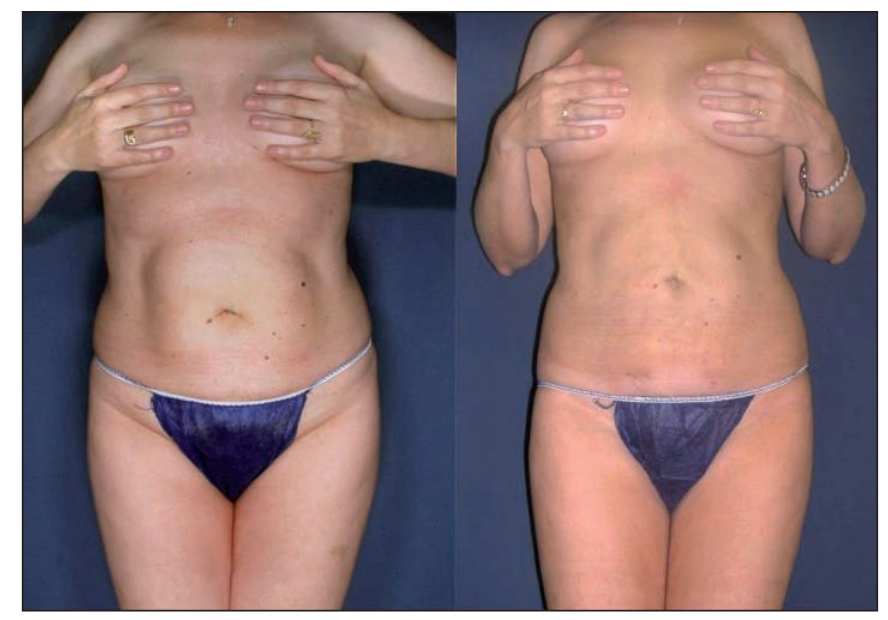

Fig. 3. Paciente de 41 años de edad con BMI 24,2. A: antes y B: 6 meses después de la cirugía. Obsérvense los buenos resultados de eliminación de la grasa abdominal y de los flancos. Notable acortamiento de las distancias supra e infraumbilicales, con desaparición de los pliegues inguinales.

La textura-flacidez de la piel comprobada antes, 1 y 6 meses después de la cirugía en las 60 pacientes, dio como valores antes del tratamiento: 16 Muy Mal (26,7\%); 23 Mal $(38,3 \%)$ y 21 Regular (35\%). En la siguiente valoración, 1 mes después del tratamiento los resultados fue-

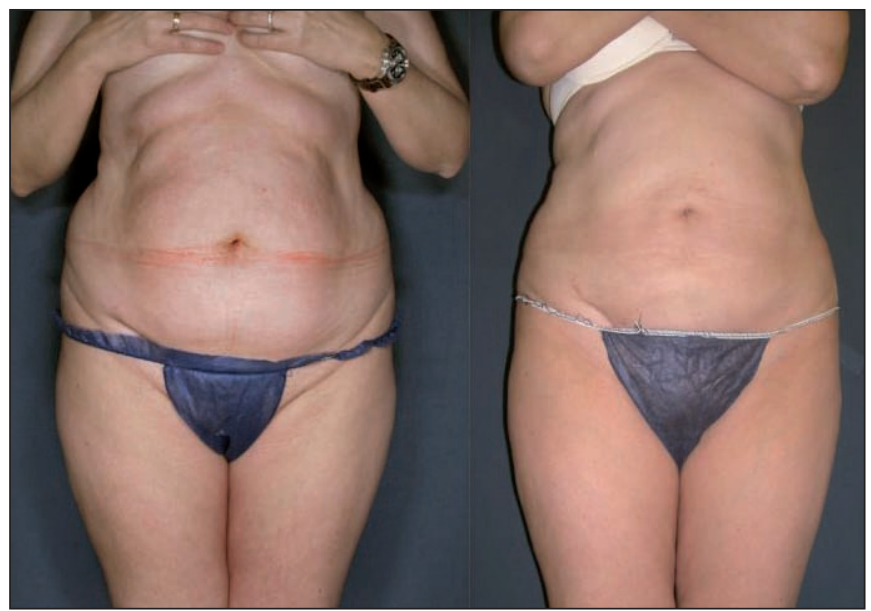

Fig. 4. Paciente de 46 años de edad con BMI de 26,4. A: antes y B: 6 meses después de la cirugía. Es evidente la disminución del volumen de la grasa del abdomen y flancos. La retracción de la piel es calificada como Muy Buena, con mejoría de la textura-flacidez. Obsérvese la desaparición de los pliegues inguinales y el acortamiento de la cicatriz de apendicetomía, que es demostrativo del "acortamiento" cutáneo conseguido por la liposucción asistida por láser.

ron: 12 Mal (20\%), Regular 27 (45\%), Bien 21 (35\%). Es decir, se observó que la calificación de piel Muy Mal había desaparecido, pasando a ser calificación Bien.

La valoración a los 6 meses fue: Regular 15 (25\%), Bien 28 (46,7\%), Muy Bien 17 (28,3\%). Lo que signifi- 
caba que a los 6 meses la mejoría de la piel fue notoria, y no se encontraron pacientes con valoración Mal o Muy Mal (Tabla II).

El test t de Student para muestras relacionadas indicó que existían diferencias significativas entre los resultados antes y 1 mes después, y entre 1 mes y 6 meses después, con $\mathrm{p}<0,05$ en ambos casos. El estudio de medias antes, 1 mes y 6 meses después fue, respectivamente, el siguiente: $1,1 \pm 0,79 ; 2,2 \pm 0,73 ; 3,0 \pm 0,74$, indicando que la mejoría de la textura-flacidez de la piel fue más notoria a los 6 meses (Fig. 5 y 6).

\section{Discusión}

El empleo de 2 láseres de Diodo con diferente emisión, 924 y 975 nm, se basa en un efecto térmico. Aunque la absorción de las mencionadas longitudes de onda se realiza por la grasa y por el agua para la emisión de 924 y $975 \mathrm{~nm}$ respectivamente, no existe selectividad de actuación porque la irradiación se realiza en modo continuo y de forma mantenida con ambos láseres. No obstante, al emplear 2 longitudes de onda se suman los efectos y la absorción de la energía láser, convertida en efecto térmico, actúa eficazmente en la formación de nuevo colágeno. Las 2 longitudes de onda logran una efectiva licuefacción de la grasa facilitando el paso de la cánula, lo que proporciona menor fatiga al cirujano, sobre todo si se realizan intervenciones de áreas extensas que incluyen la región superior e inferior del abdomen y ambos flancos. El efecto térmico del láser consigue también cerrar los pequeños vasos sanguíneos, reduciendo la pérdida hemática hasta en un 50\% en comparación con la liposucción convencional (13).

Entre las ventajas mencionadas, asociadas al depósito térmico de los 2 láseres, se suman los efectos notables en la retracción de la piel según los resultados de este estudio y de acuerdo también con otros autores (3). La cámara digital de superficie constituye un método de diagnóstico comparativo fiable y objetivo de evaluación de la textura-flacidez cutánea $(14,15)$. Weiss en 2009 (16), y nosotros mismos en 2012 y $2014(7,17)$, hemos comunicado sobre los buenos resultados alcanzados por la lipólisis láser para la remodelación de rodillas y pantorrillas, observando una alta satisfacción por parte de las pacientes tratadas.

De acuerdo a la literatura, el láser de $980 \mathrm{~nm}$ de emisión es eficaz como ayuda a la liposucción (6). Esta observación ha sido corroborada en estudios posteriores (8) sobre tratamiento de la ginecomastia, asistida por láser de Diodo de $1470 \mathrm{~nm}$. En el estudio de referencia se validó la reducción del tejido adiposo mediante ecografía, constatando la reducción del perímetro torácico y del diámetro de las areolas, indicativo este último del efecto de retracción de la piel gracias al depósito térmico del láser.

Tabla II. Progresión de los resultados obtenidos por evaluación con cámara digital de superficie en la mejoría de la textura-flacidez de la piel, 1 mes y 6 meses después de practicada la cirugía

\begin{tabular}{|l|c|c|c|c|c|}
\hline \multicolumn{1}{|c|}{ Resultados } & Muy Mal & Mal & Regular & Bien & Muy Bien \\
\hline Antes de la cirugía & $16(26,7 \%)$ & $23(38,3 \%)$ & $21(35 \%)$ & & \\
\hline 1 mes después & & $12(20 \%)$ & $27(45 \%)$ & $21(35 \%)$ & $28(46,7 \%)$ \\
\hline 6 meses después & & & $15(25 \%)$ & $17(28,3 \%)$ \\
\hline
\end{tabular}

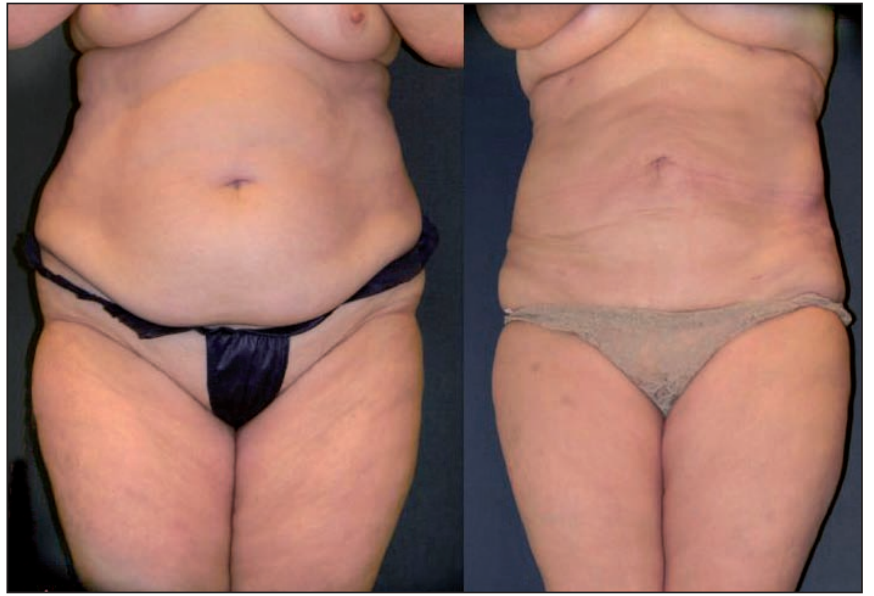

Fig. 5. Paciente de 52 años de edad con BMI de 26,8. A: antes y B: 6 meses después de la cirugía. Buenos resultados con evidente eliminación de la grasa de abdomen y flancos. La textura-flacidez cutánea es buena, con notable contracción.

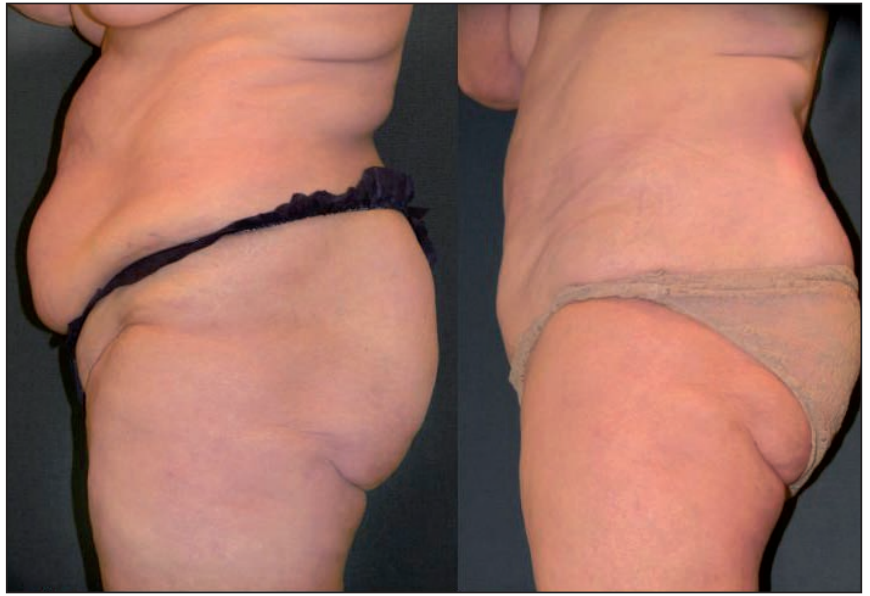

Fig. 6. La misma paciente A: antes y B: 6 meses después de la cirugía. Obsérvese el aplanamiento del abdomen en la vista de perfil. La piel se ha retraído y los flancos se presentan libres de grasa y sin el pliegue de la piel que se mostraba antes de la cirugía. 
En el mismo trabajo, los autores concluyeron que no influye tanto la longitud de onda como la energía total acumulada en el área quirúrgica, en concordancia con las investigaciones de Mordon $(18,19)$.

Respecto al dolor secundario a la cirugía, los estudios realizados indican que este síntoma $(6,7,20)$ suele controlarse bien con paracetamol de 1 gr. 3 veces al día durante 5 días. Estas observaciones coinciden con el estudio de Llanos (21) en cuanto a que las pacientes experimentan menos dolor en las liposucciones asistidas por láser que en las liposucciones convencionales. En nuestro estudio, la indicación de $10 \mathrm{mg}$ diarios de prednisona por vía oral durante 2 semanas tuvo como finalidad evitar una gran reacción inflamatoria que pudiera repercutir en una fibrosis postquirúrgica excesiva.

La diferencia en las valoraciones subjetiva y objetiva, a pesar de emplear la misma escala GAIS, tanto por las pacientes como por el médico, es atribuible al hecho de que las pacientes suelen solo valorar el resultado estético sin atender a variables como la diástasis de rectos y la ex-

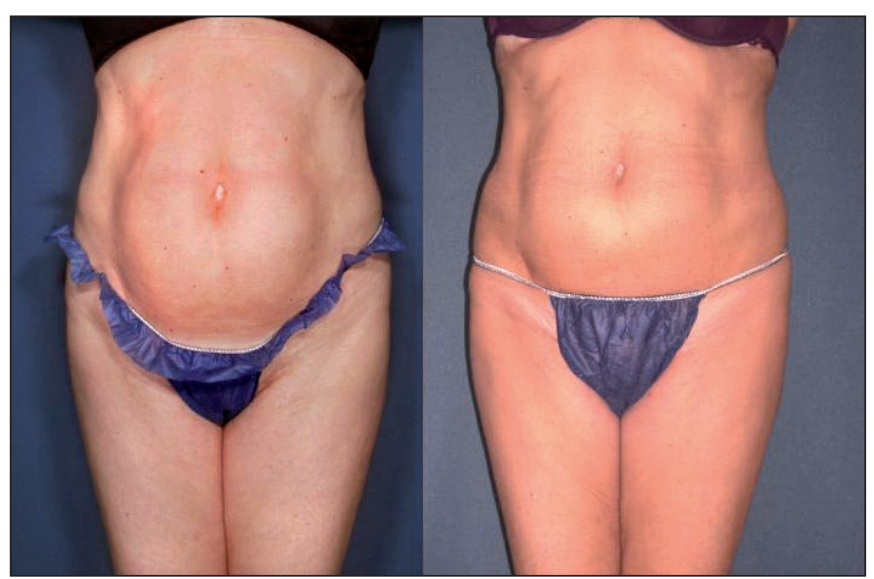

Fig. 7. Paciente de 44 años con BMI de 24 con diástasis de rectos abdominales de acuerdo a la exploración ecográfica realizada antes de la intervención. A: antes y B: 6 meses después de la cirugía. El abdomen y los flancos muestran clara diferencia en cuanto a la eliminación de la grasa. Se observa también un resultado calificado como Muy Bueno en la mejoría de la textura-flacidez cutánea; no obstante, la paciente no estaba satisfecha por encontrarse el abdomen abultado y no plano.

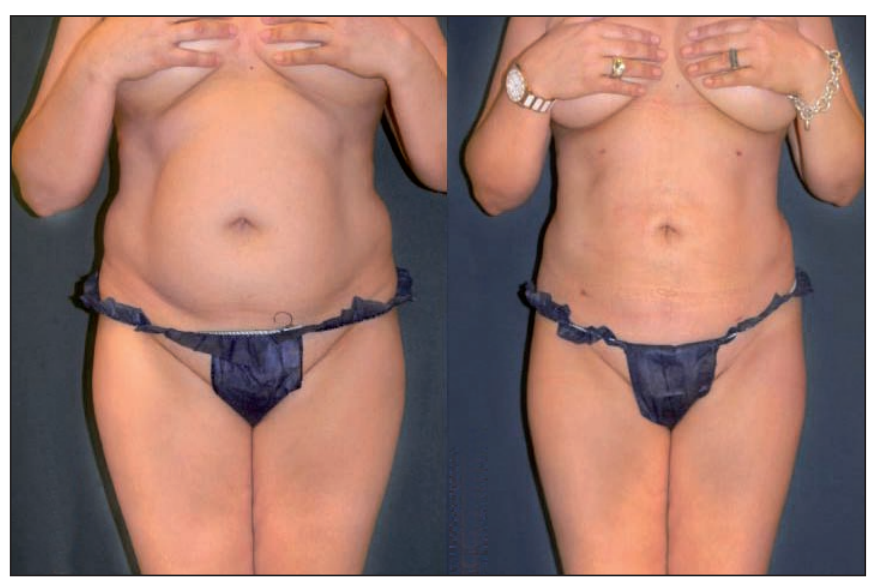

Fig. 9. Paciente de 43 años de edad con BMI de 25,4. A: antes y B: 6 meses después de la cirugía. El resultado es calificado como Muy Bueno en cuanto a la eliminación de grasa del abdomen y de los flancos. La piel redundante y flácida ha mejorado. celente retracción de la piel que se consigue. De ahí la correlación entre los resultados valorados por el médico comparativamente a la valoración de las pacientes. Sin embargo, la valoración subjetiva de resultados Buenos y Muy Buenos alcanzó al 57,1\% de las pacientes (194), aunque todas ellas eran mayores de 40 años, multíparas (2,4 \pm 0,48 embarazos), 141 (41,47\%) pacientes habían sufrido al menos una cesárea, y $310(91,18 \%)$ presentaban diástasis de rectos en grado severo o moderado (Fig. 7 y 8 ).

A estas pacientes con diástasis se les recomendó someterse a una abdominoplastia para reparar la separación de la pared del abdomen, pero se negaron en todos los casos a aceptar la recomendación. En el estudio que nos ocupa es preciso mencionar este detalle porque las pacientes que presentaban mayor dimensión de la diástasis de rectos abdominales manifestaron estar más descontentas con los resultados, aunque la valoración objetiva de textura-flacidez hubiera sido máxima. Esto ocurrió a pesar de haber informado a las pacientes de que la pre-

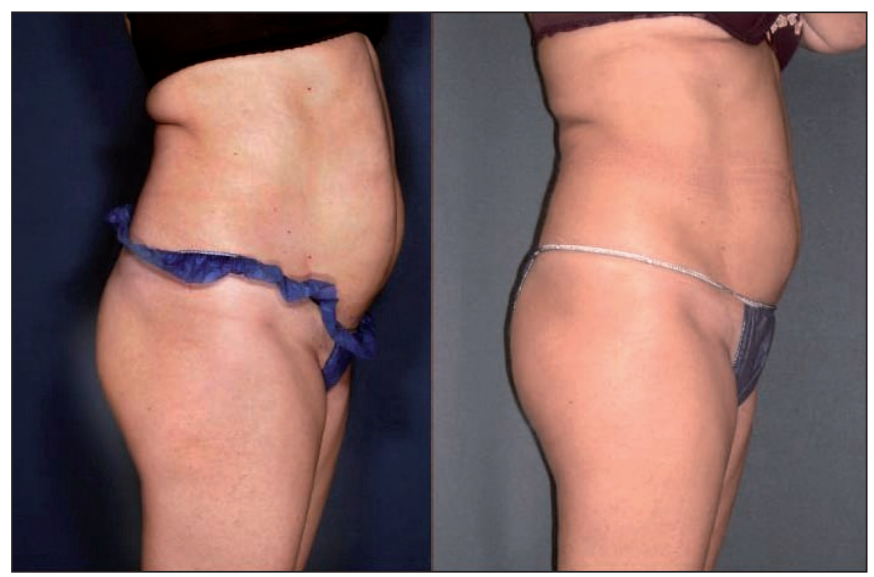

Fig. 8. La misma paciente A: antes y B: 6 meses después de la cirugía. La vista de perfil permite observar la falta de aplanamiento del abdomen después de la intervención, aunque la grasa acumulada en el abdomen ha sido eliminada y la flacidez de la piel ha mejorado por la liposucción asistida por láser.

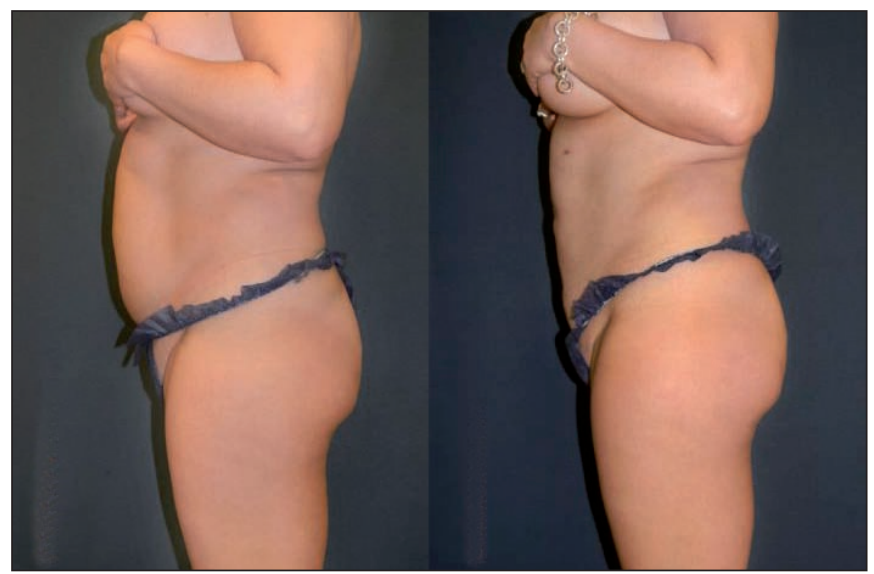

Fig. 10. La misma paciente en la vista de perfil. La eliminación de la grasa y la contracción de la piel en calificada como Muy Buena 6 meses después de la cirugía. 
sencia de diástasis no permitiría mejorar la pronunciación y abombamiento de la forma del abdomen incluso aunque objetivamente se demostrase que se había logrado eliminar significativamente la capa y los depósitos localizados de tejido graso. Si bien todas las pacientes comprendieron los límites de la cirugía explicados antes de su realización, una vez efectuada la misma olvidaron su expresa aceptación de los términos y pronóstico de la misma. No obstante los mencionados inconvenientes, estimamos que la liposucción asistida por láser es una opción válida para eliminar tejido adiposo y conseguir una notable retracción cutánea. La contracción de la piel redundante sigue una correlación directa con la edad de las pacientes, lo que no es sorprendente si se tiene en cuenta que el factor edad es importante cuando se trata de formar nuevo colágeno (Fig. 9 y 10).

\section{Conclusiones}

La liposucción asistida por láser es una técnica que progresivamente se afianza en la práctica quirúrgica por presentar varias ventajas sobre la liposucción convencional: la licuefacción de la grasa por efecto de la energía térmica depositada en el área tratada facilita la lipoaspiración; las maniobras de irradiación térmica del láser son capaces de romper los tractos fibrosos localizados en las adiposidades, haciendo que el trabajo del cirujano sea menos laborioso, sobre todo en aspiraciones de grandes volúmenes; la coagulación de los pequeños vasos sanguíneos que logra el láser, reduce la pérdida hemática propia de la cirugía; el láser estimula de la formación de colágeno mejorando la textura y combatiendo la flacidez de la piel; finalmente, debido al menor trauma quirúrgico, la recuperación postoperatoria es más rápida y con menor morbilidad, lo que sin duda repercute en el grado de satisfacción que muestran las pacientes.

Creemos que es notable también cómo el láser permite obtener resultados más homogéneos de la piel disminuyendo la presencia de irregularidades y depósitos de grasa residuales.

Importante señalar que estos resultados mejoran cuando las pacientes presentan depósitos de grasa localizada y no sufren diástasis de los rectos abdominales como consecuencia de partos múltiples. También es importante que, de forma ideal, las pacientes no presenten un índice de masa grasa corporal superior a 26 para poder predecir y obtener óptimos resultados.

\section{Declaración}

Los autores manifiestan no tener ningún interés comercial con los productos o aparatos reseñados en el presente estudio, y agradecen a D. Juan Carlos Medina, estadístico, su colaboración en el análisis de datos.

\section{Dirección del autor}

\author{
Dr. Javier Moreno Moraga \\ Instituto Médico Láser \\ Paseo General Martínez Campos 33 \\ 28010 Madrid. España \\ e-mail: jmorenomoraga@iml.es
}

\section{Bibliografía}

1. Licata G, et al.: Lipolysis using a new 1540 -nm diode laser: a retrospective analysis of 230 consecutive procedures. J Cosmet Laser Ther. 2013; 15(4):184-192.

2. Sasaki GH.: Quantification of human abdominal tissue tightening and contraction after component treatments with 1064-nm/1320-nmlaser-assisted lipolysis: clinical implications. Aesthet Surg J. 2010; 30(2):239-245.

3. Weiss RA, Beasley K.: Laser-assisted liposuction using a novel blend of lipid- and water-selective wavelengths. Lasers Surg Med. 2009; 41(10):760-766.

4. Moreno-Moraga J, Royo J.: Our experience treating 500 lipodystrophic areas with the SlimLipo Laser ${ }^{\mathrm{TM}}$, a new device that induces lipolysis and heating of the dermis and fibrous septa of fatty tissue. J Clin Dermatol 2010; 1(2):1-10.

5. Di Bernardo BE.: Randomized, blinded split abdomen study evaluation skin shrinkage and skin tightening in laser-assisted liposuction versus liposuction control. Aesthet Surg. 2010; 30(4):593-602.

6. Leclère FM, Trelles M, Moreno-Moraga J, Servell P, Unglaub F, Mordon SR.: 980-nm laser lipolysis (LAL): about 674 procedures in 359 patients. J Cosmet Laser Ther. 2012; 14(2):67-73.

7. Leclère FM, Moreno-Moraga J, Mordon S, Servell P, Unglaub F, Kolb F, et al.: Laser-assisted lipolysis for cankle remodeling: a prospective study in 30 patients. Lasers Med Sci. 2014; 29(1):131-136.

8. Trelles MA, Alcolea JM, Bonanad E, Moreno-Moraga J, Leclère FM.: Liposucción laser-asistida en ginecomastia: seguimiento ecográfico y estadístico de la retracción cutánea. Cir plást iberolatinoam. 2013; 39(4): 425-438.

9. Badin AZ, Moraes LM, Gondek L, Chiaratti MG, Canta L.: Laser lipolysis: flaccidity under control. Aesthetic Plast Surg. 2002; 26(5):335-339.

10. Klein JA.: The tumescent technique for liposuction surgery. Am J Cosmet Surg. 1987; 4:263-267.

11. Klein JA.: The tumescent technique. Anesthesia and modified liposuction technique. Dermatol Clin. 1990; 8(3): 425-437.

12. Trelles M, Bonanad E, Moreno-Moraga J, Alcolea J, Mordon S, Leclère FM.: Lipólisis láser y liposucción en ginecomastia: retracción cutánea eficaz y segura. Rev Col Bras Cir. 2013; 40(1):23-31.

13. Abdelaal MM, Aboelatta YA.: Comparison of Blood Loss in Laser Lipolysis vs Traditional Liposuction. Aesthet Surg J. 2014; 34(6):907-912.

14. Weiss ET, et al.: Three-dimensional surface imagining for clinical trials: improve precision and reproducibility in circumference measurement of thighs and abdomens. Lasers Surg Med. 2009; 41(10):767-773.

15. Regula CG, Lawrence N.: Update o liposuction: laserassisted liposuction versus tumescent liposuction. Curr Derm Rep. 2014; 3:127-134.

16. Moreno-Moraga J, Trelles MA, Mordon S, Unglaub F, 
Bravo E, Royo de La Torre J, et al.: Laser-assisted lipolysis for knee remodeling: a prospective study in 30 patients. J Cosmet Laser Ther. 2012; 14(2):59-66.

17. Mordon S, Plot E.: Laser lipolysis versus traditional liposuction for fat removal. Expert Rev Med Devices. 2009; 6(6):677-688.

18. Mordon S, Eymard-Maurin AF, Wassmer B, Ringot J.: Histologic evaluation of laser lipolysis: pulsed 1064nm Nd:YAG laser versus cw 980-nm diode laser. Aesthetic Surg J. 2007; 27(3):263-268.

19. Chia CT, Theodorou SJ.: 1,000 consecutive cases of laser-assisted liposuction and suction-assisted lipectomy managed with local anesthesia. Aesthetic Plast Surg. 2012; 36(4):795-802.
20. Llanos S, et al.: Comparación del dolor secundario a lipoaspiración tradicional versus lipólisis láser. Estudio prospectivo. Cir plást iberolatinoam. 2007; 33(4):221232.

21. McBean JC, Katz BE.: A pilot study of the efficacy of a 1,064 and 1,320 $\mathrm{nm}$ sequentially firing $\mathrm{Nd}$ :YAG laser device for lipolysis and skin tightening. Lasers Surg Med. 2009; 41(10):779-784. 